\title{
Comparing the rate of growth and metabolic efficiency of yeast experiencing environmental stress or genetic damage*
}

\author{
Kacper Sendra\#, Agata Jakubowska and Ryszard Korona ${ }^{\bowtie}$ \\ Institute of Environmental Sciences, Jagiellonian University, Kraków, Poland
}

Physical stresses, toxic substances, and mutations can cause marked decline in the rate of growth (RG). We report that the efficiency of growth (EG), i.e. converting glucose into biomass, responds less profoundly. It remains nearly unaffected for some physical and chemical stresses, but decreases substantially for others, specifically those affecting membrane integrity or ion homeostasis. Mutations (gene deletions) can heavily reduce RG, but much less EG. Moreover, there is no apparent relation between the function of deleted gene and EG. Generally, assays of EG appear as more laborious, less precise, and less informative than those of RG.

Key words: fitness cost, growth rate, metabolic efficiency, environmental stress, chemical stress, gene deletion

Received: 09 October, 2013; 07 December, 2013; accepted: 19 December, 2013; available on-line: 27 December, 2013

\section{INTRODUCTION}

The construction of a gene-deletion collection allowed to test the impact of hundreds of physical and chemical stress factors on thousands of yeast strains (Giaever et al., 2002, Steinmetz et al., 2002, Hillenmeyer et al., 2008, Blomberg 2011). All these studies were based on the assumption, that malfunctioning of a deletion strain in a particular environment is a sign of the deleted gene's normal usage in it. Although specific experimental arrangements differ, most studies rely on one phenotypic trait, the rate of growth (RG). Efficiency of growth (EG), defined as the efficiency of converting resources into biomass, could be another one. There were few attempts to estimate EG for the whole collection of yeast gene deletions. In those studies, however, neither the production of biomass nor consumption of glucose were actually measured. Instead, only the amount of biomass was estimated, and not directly but through measuring the optical density (OD) of cultures in stationary phase. One study of this type found such data much less useful than those relating to RG (Warringer et al., 2003), while another research suggested, that they can be informative (Bell, 2010). In both these studies, however, actual amount of consumed resources was unknown. This is because even if all deletion strains consumed glucose completely, fast-growing strains had more time to metabolize ethanol (and thus add biomass) than slow-growers before OD was measured. Moreover, OD is only an indirect estimate of biomass and is sensitive to the morphology of cells, for example, their size. The yeast deletion strains differ substantially in cell morphology, and therefore, comparison of OD of different strains can be misleading (Jorgensen et al., 2002).
We consider it more appropriate to work with cells growing exponentially in glucose-rich media. This stands to reason that in such media glucose is a sole source of energy, because even though ethanol accumulates, it is not metabolized due to strong allosteric and transcriptional repression of oxidative metabolism (Zaman et al., 2008). Metabolic/energetic expenses of such cells can be accurately approximated by measuring the rate of glucose uptake. A reliable method of estimating the yield of biomass is to weigh dry mass of cells. However, assays of glucose consumption and dry mass production are laborious,. They were not done for the whole collection of gene-deletions. In our recent study of epistasis, we have got estimates of the biomass production per consumed glucose for a sample of strains with a different number of gene deletions (Jakubowska \& Korona, 2012). The estimates of biomass were somewhat improved when compared with previous studies, but still imperfect. We have found that strains with single gene deletions differ in EG, but the differences are probably modest. In the work described here, we use a sample of deletion strains to estimate both RG and EG possibly most accurately. We also asked how RG and EG change when not deletions of genes but external stresses are present. In this ways, we wanted to test how the changes in RG and EG caused by mutations compare to those imposed by unfavorable environmental conditions.

\section{MATERIALS AND METHODS}

Strains and media. The strain used to test the impact of chemical and physical factors was BY4742 MATa leu2 bis3 ura3 hys2. It is the strain in which complete collection of yeast gene deletions was engineered (Giaever et al., 2002).

Growth media consisted of YPD (1\% yeast extract, $2 \%$ peptone, $2 \%$ glucose) supplemented with chemicals at defined concentrations. Test media were obtained by mixing double-concentrated YPD with equal aliquots of water with fresh solutions of test chemicals prepared at concentrations twice higher than final ones. Stock solutions were kept in water or, in case of nystatin and benomyl, in DMSO. The agents and their modes of action are listed in Table 1. Specific concentrations used in this study were as follows: benomyl $(\mu \mathrm{g} / \mathrm{ml}) 15,28$,

e-mail: ryszard.korona@uj.edu.pl

*Presented at the 3-rd Workshop on Microbiology "MIKROBIOT 2013" in Łódź, Poland.

\#Present address: Institute for Cell and Molecular Biosciences, The Medical School, Newcastle University, Catherine Cookson Building, Framlington Place, Newcastle upon Tyne, NE2 4HH, Great Britain Abbreviations: RG, rate of growth; $E G$, efficiency of growth; $O D$, optical density 
40; caffeine $(\mathrm{mg} / \mathrm{ml})$ 0.80, 1.35, 2.50; cold $\left({ }^{\circ} \mathrm{C}\right) 25,22$, 18; $\mathrm{CuSO}_{4}(\mathrm{mg} / \mathrm{ml})$ 2.0, 2.5, 2.8; cycloheximide $(\mathrm{ng} / \mathrm{ml})$ 20, 80, 120; DMSO (\%) 5.0, 7.5, 8.0; ethidium bromide $(\mu \mathrm{g} / \mathrm{ml}) 30,55,85 ; \mathrm{KCl}(\mathrm{mg} / \mathrm{ml}) 70,120,140$; methanol (\%) 5.0, 7.0, 8.5; $\mathrm{NaCl}(\mathrm{mg} / \mathrm{ml}) 35,60,80$; paromomycin $(\mathrm{mg} / \mathrm{ml}) \quad 0.2,0.4,0.9 ; \mathrm{ZnSO}_{4}(\mathrm{mg} / \mathrm{ml}) \quad 0.65,1.00,1.50$. As explained in Results, some factors were tested at one level only: benzoic acid $(\mathrm{mg} / \mathrm{ml}) 1.0$; diamide $(\mathrm{mg} / \mathrm{ml})$ 0.25; EDTA (mg/ml) 0.1; high $\mathrm{pH}(\mathrm{pH})$ 9; hygromycin B $(\mu \mathrm{g} / \mathrm{ml}) 20$; nalidixic acid $(\mathrm{mg} / \mathrm{ml}) 0.5$; nystatin $(\mu \mathrm{g} /$ $\mathrm{ml})$ 1.4; SDS $(\mathrm{mg} / \mathrm{ml})$ 0.35; sodium salicylate $(\mathrm{mg} / \mathrm{ml})$ 25; sorbitol $(\mathrm{mg} / \mathrm{ml}) 380$.

Gene deletions. A sample of about two hundred strains was tested for the efficiency of converting glucose into biomass in our former study (Jakubowska, Korona, 2012). Those measurements relied on OD scores corrected for the size of cells only, and therefore, are indirect estimates of biomass only. Here, we carried out direct measurements of growth rate, glucose consumption, and dry mass for a sample of 20 gene deletions. The 20 gene deletions were selected as those promising to show substantial decrease in EG. The deleted genes included: TPM1, actin assembly; GND1, response to oxidative stress; IMG2, mitochondrial translation; ELP3, transcription; LRP1, RNA metabolism; $M R H 4$, ribosome biogenesis; TAF14, chromatin remodeling; RAD54, DNA translocase; VID22, protein catabolism; DSS1, RNA metabolism; $C O Q 3$, ubiquinone synthesis; $\mathrm{RAD6}$, DNA ligase; BUB1, cell cycle; $A I M 10$, proline-tRNA ligase; RPL $8 A$, translation; MET7, methionine biosynthesis; REG1, carbohydrate metabolism; SSN8, transcription; PMR1, calcium transport ATPase; HUR1, DNA replication. Functional descriptions are borrowed from the Saccharomyces Genome Database (www.yeastgenome.org).

Growth rate, glucose consumption, dry mass. Experimental cultures were initiated by inoculating $25 \mu \mathrm{l}$ of overnight culture into $10 \mathrm{ml}$ of fresh medium. The new cultures were kept in vials of $30 \mathrm{~mm}$ in diameter, loosely capped, and agitated at $250 \mathrm{rpm}$. Temperature was set to $30^{\circ} \mathrm{C}$ except for the cold stress gradient. After reaching $\mathrm{OD}_{600}$ of about 0.1 , aliquots of $200 \mu \mathrm{l}$ were taken every $1 \mathrm{~h}$ and their density was measured. Because OD readings showed a very good fit to the exponential growth curve for ODs between 0.1 and 0.4 , OD scores within this interval were log-normal transformed, and used to calculate the rate of growth (RG).

Growth of the cultures was terminated when $\mathrm{OD}_{600}$ was between 0.4 and 0.5 by transferring the vials to ice water and vigorous shaking. A sample of $5 \mathrm{ml}$ was taken from every culture and spun down; pellet was preserved for further assays of dry mass; a sample of supernatant was saved to assay glucose concentration. The latter was done by diluting supernatant 1:200 and applying a kit based on an enzymatic reaction and fluorescence reading (D-GLUCOSE-HK, Megazyme). Pellets obtained from the $5 \mathrm{ml}$ samples were washed three times, vacuum dried, and then left loosely capped for 5 days in a drier set to $65^{\circ} \mathrm{C}$. The final weight of the dried pellets was a few milligrams, they were weighed with an accuracy of one hundred of a milligram.

All measurements were replicated three times, that is, three independent cultures were initiated and their maximum growth rate, glucose consumption, and dry mass production were individually estimated. One replica of the diamide treatment was lost.

\section{RESULTS}

\section{Environmental stresses}

We started with 12 chemical or physical agents for which we were able to obtain incremental deceleration of growth by about $1 / 4,1 / 2$, and $3 / 4$. Doses required for these defined effects were found in pilot experiments. Figure 1 shows what were the effects of specific agents on RG and EG. A quadratic regression line was fitted in every case to show a general trend. Gradients of stress could not be completed for 10 agents. A chief rea-
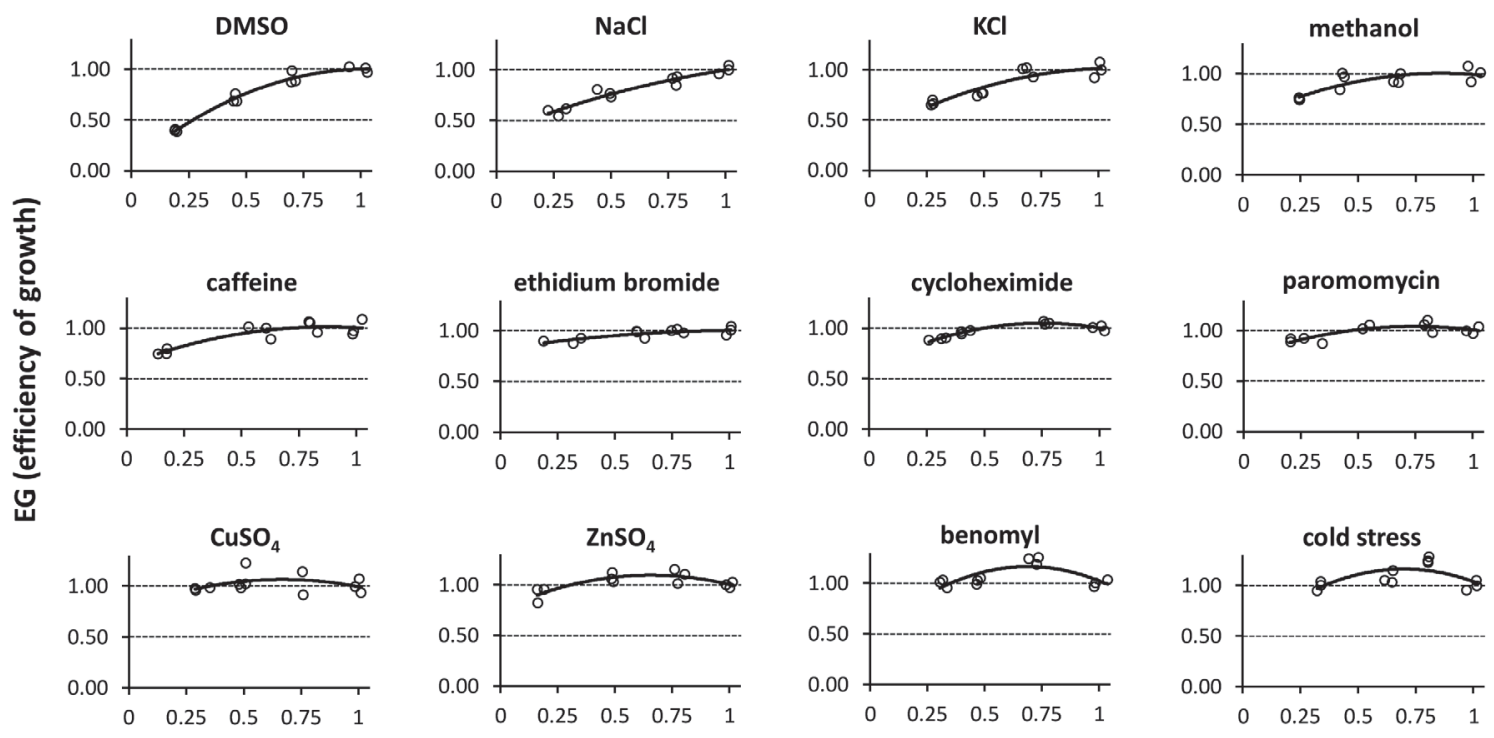

\section{RG (rate of growth)}

Figure 1. Efficiency of growth (EG) along stress gradients.

Horizontal axes: normalized rate of growth (RG). Vertical axes: normalized EG, that is the ratio of dry mass over consumed glucose. Both variables were normalized by dividing them respectively by average RG and EG in non-stressful environment. The doses of stress factors reducing RG to $0.75,0.5$ and 0.25 are listed in Materials and Methods. 


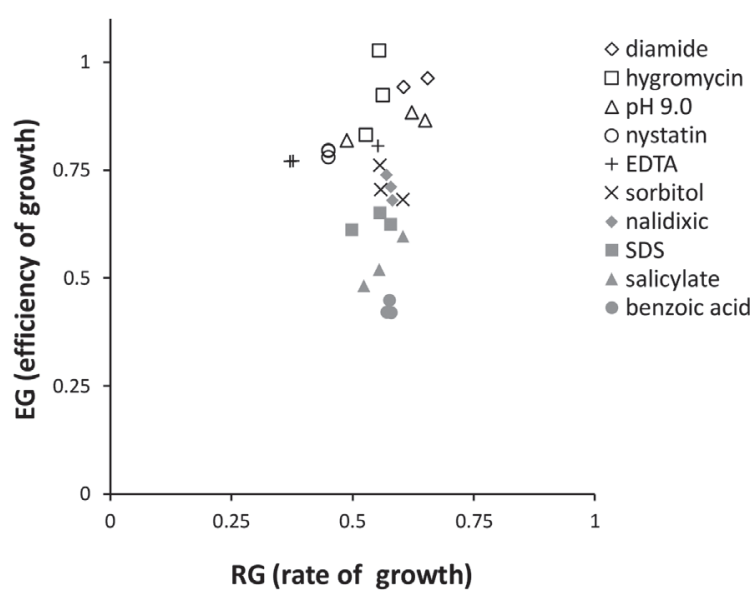

Figure 2. Efficiency of growth (EG) under single-intensity stresses.

Axes and normalization as described in Fig. 1.

son was a precipitous decrease in RG at narrow regions of stress intensities. Moreover, the critical doses of stress factors were apparently very much affected by even slight fluctuations in the preparation of stocks, working media, and conditions of culturing. In particular, growth curves became irregular making calculation of RG unre-

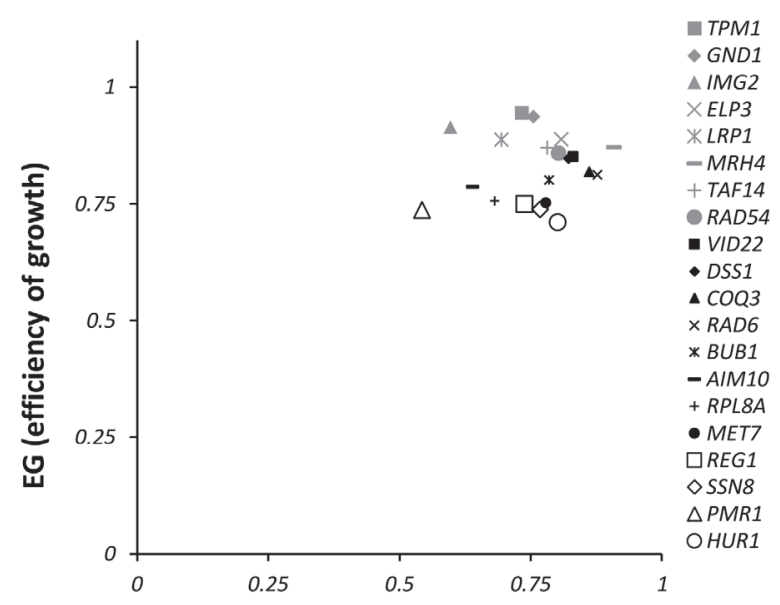

RG (rate of growth)

Figure 3. Efficiency of growth (EG) of gene deletions strains.

Means instead of three individual estimates per gene deletion are shown. Axes as described in Fig. 1. Normalization was done by dividing $R G$ and $E G$ of deletion strains by $R G$ and $E G$ of the strain in which deletions were done. RG and EG were measured in nonstressful environment. Gene functions are listed in Materials and Methods.

\section{Table 1. Stress agents.}

\begin{tabular}{|c|c|c|}
\hline Agent & Mode of action & Reference \\
\hline Benomyl & cytoskeleton depolimerization & (Hoyt et al., 1990) \\
\hline benzoic acid & destabilization of membrane proton gradient & (Verduyn et al., 1992) \\
\hline Caffeine & disturbance of growth signaling pathways & (Wanke et al., 2008) \\
\hline cold & ineffective assembly of protein complexes & (Guthrie et al., 1969) \\
\hline $\mathrm{CuSO}_{4}$ & oxidative stress & (Yasokawa et al., 2008) \\
\hline cycloheximide & inhibition of protein synthesis & (Stocklein Piepersberg, 1980) \\
\hline Diamide & oxidative stress & (Wemmie et al., 1997) \\
\hline DMSO & decreased cell membrane integrity & (Murata et al., 2003) \\
\hline EDTA & increased cell wall porosity & (de Nobel, 1989) \\
\hline ethidium bromide & inhibition of DNA and RNA polimerization & (Newton, 1957) \\
\hline high pH & alkaline stress, ionic imbalance & (Serrano, et al., 2004) \\
\hline hygromycin B & interference with translation elongation & (Chuang \& Madura, 2005) \\
\hline $\mathrm{KCl}$ & osmotic stress & (Arino et al., 2010) \\
\hline Metanol & destabilization of cell membrane structure & (Yasokawa et al., 2010) \\
\hline $\mathrm{NaCl}$ & osmotic stress, $\mathrm{K}+/ \mathrm{Na}+$ imbalance & (Arino et al., 2010) \\
\hline nalidixic acid & inhibition of topoisomerase & (Ludewig et al., 1994) \\
\hline Nystatin & increased cell membrane parmeability & (Venables \& Russell, 1975) \\
\hline paromomycin & decreased fidelity of translation & (Balasundaram et al., 1999) \\
\hline SDS & damaged cell membrane & (Sirisattha, et al., 2004) \\
\hline sodium salicylate & acidification of cytoplasm & (Giardina Lis, 1995) \\
\hline Sorbitol & osmotic stress & (Hirasawa et al., 2006) \\
\hline $\mathrm{ZnSO}_{4}$ & interference with ion-binding proteins & (Pagani et al., 2007) \\
\hline
\end{tabular}


liable. For these stresses, we decided to concentrate on single doses, which would be intense enough to cause about $50 \%$ reduction of RG. Fig. 2 shows RGs and EGs for these agents.

Figures 1 and 2 show that EG varied depending on what factor was tested. This is especially well visible in Fig. 1, where some stress factors nearly do not change EG even though RG is four times lower than under no-stress conditions, while other stresses lead to pronounced decreas in EG. In case of the single-intensity stresses (Fig. 2) the reaction of EG at the $0.5 \mathrm{RG}$ was on average stronger than that in the gradients. In sum, the two used measures of stress intensity, RG and EG, can give considerably contrasting patterns of reaction to eternal stress. Under most conditions tested here, RG was sensitive and monotonic in its reaction to increasing stress intensity. The reaction of EG was neither equally universal nor comparably strong.

\section{Gene deletions}

Figure 3 shows EGs and RGs for 20 gene deletions. None of the deletions reduced EG as much as the most damaging stresses shown in Figs. 1 and 2. Reduction in RG was also less pronounced than under the most severe eternal stresses. It was already noted, however, that the 20 deletions were chosen from a sample of about 200 strains as those likely to have especially large negative effects on EG, not RG.

\section{DISCUSSION}

Our experiments demonstrate that two measures of reaction to stress, RG and EG, can give considerably different results. Under most environmental conditions tested here, RG declined strongly as the intensity of stress increased. The efficiency of converting glucose into biomass was not equally sensitive. Under some stresses EG decreased significantly, but there were also stresses in which hardly any change was seen. Comparison of Figs. 1 and 2 with Table 1 leads to a conclusion, that the most damaging to EG were those factors, which affected membrane gradients, or caused strong osmotic stresses. Intense rebuilding of the gradients and deep restructuring of the cell metabolism under high osmotic pressure are known to require large amounts of energy (Hohmann, 2002). Factors that did not affect EG, or did it only marginally, were more diverse. Some were very specific or had narrow targets. For example, aminoglycoside antibiotics slow down the process of translation; however, they do not destroy its key and costly machinery, namely, the ribosome subunits. Other, as divalent metal ions, tend to outcompete other ions in binding specific proteins, but in this experiment their concentration was generally low, and thus they did not cause the osmotic stress or strong ionic disequilibrium.

Regarding mutational damage, our present assays confirm earlier observations, that mutations can cause a loss in EG. The sample of gene deletions used in this study was not random, but chosen as those expected to result in an especially strong negative impact on EG. But, even though these were the deletions most damaging to EG, their absolute effects were rather moderate when compared with those accompanying external stresses. The sample size was rather small, but the tested genes were substantially different from each other in terms of functions they coded for (Materials and Methods). Unlike stresses, which turned out to be especially damaging when membrane integrity was affected, the gene dele- tion with highest EG effects had no apparent common (prevailing) function. This suggests that low EG is only one of multiple phenotypes affected by deletion of these pleiotropic genes, and therefore its applicability to functional analyses may be limited. This finding, and the fact that the effects of gene deletions on $E G$ were rather low, questions the rationale for using EG estimates for phenotypic assays of large collections of mutants. The reason is that measurements of EG are both much more laborious and less precise than that of RG. A question arises, how much information is lost or misinterpreted when EG is not assayed. This could be important for the assays in which the rate of growth of multiple strains is measured simultaneously on a common agar plate. The eventual differences in the colony size are affected both by RG and EG, but it is common to ignore the impact of EG (Blomberg, 2011). We think that this practice is likely allowable because EG can have any impact only when colonies are large enough to use non-trivial share of glucose present in agar. Furthermore, as our results suggest, even when the differences in EG are present, their final effect would be relatively weak.

In our earlier study, we have pointed out that some mutations can lead to a substantial decrease in the rate of growth, but not in the metabolic efficiency (Jakubowska \& Korona, 2012). This was confirmed in the present study (Fig. 3). However, although there was no general tendency that the metabolic efficiency declined with the rate of growth, there were mutations which were evidently deleterious to the efficiency. Considering environmental stresses, their effects on the efficiency were similarly different: some environments had a clearly negative impact while others appeared completely neutral. The impact of stresses on the efficiency is partly understood, at least for some environments (Hohmann, 2002). The question why only some mutations make the metabolism inefficient is well worth asking and can potentially lead to deeper understanding of the cell physiology.

\section{Financial support}

This work was supported by a grant from the Institute of Environmental Sciences, Jagiellonian University, no. DS/WBiNoZ/INoS/762.

\section{REFERENCES}

Arino J, Ramos J, Sychrova H (2010) Alkali metal cation transport and homeostasis in yeasts. Microbiol Mol Biol Rev 74: 95-120.

Balasundaram D, Tabor CW, Tabor H (1999) Sensitivity of spermidine-deficient Saccharomyces cerevisiae to paromomycin. Antimicrob Agents Chemother 43: 1314-1316.

Bell G (2010) Experimental genomics of fitness in yeast. Proc Biol Sci 277: 1459-1467.

Blomberg A (2011) Measuring growth rate in high-throughput growth phenotyping. Curr Opin Biotechnol 22: 94-102.

Chuang SM, Madura K (2005) Saccharomyces cerevisiae Ub-conjugating enzyme Ubc4 binds the proteasome in the presence of translationally damaged proteins. Genetics 171: 1477-1484.

de Nobel JG, Dijkers C, Hooijberg E, Klis FM (1989) Increased cell wall porosity in Saccharomyces cerevisiae after treatment with dithiothreitol or EDTA. J General Microbiol 135: 2077-2084.

Giaever G, Chu AM, Ni L et al. (2002) Functional profiling of the Saccharomyces cerevisiae genome. Nature 418: 387-391.

Giardina C, Lis JT (1995) Sodium salicylate and yeast heat shock gene transcription. I Biol Chem 270: 10369-10372.

Guthrie C, Nashimoto H, Nomura M (1969) Structure and function of E. coli ribosomes. 8. Cold-sensitive mutants defective in ribosome assembly. Proc Natl Acad Sci USA 63: 384-391.

Hillenmeyer ME, Fung E, Wildenhain J et al. (2008) The chemical genomic portrait of yeast: uncovering a phenotype for all genes. Science 320: 362-365.

Hirasawa T, Ashitani K, Yoshikawa K et al. (2006) Comparison of transcriptional responses to osmotic stresses induced by $\mathrm{NaCl}$ and 
sorbitol additions in Saccharomyces cerevisiae using DNA microarray. I Biosci Bioeng 102: 568-571.

Hohmann S (2002) Osmotic stress signaling and osmoadaptation in yeasts. Microbiol Mol Biol Rev 66: 300-372.

Hoyt MA, Stearns T, Botstein D (1990) Chromosome instability mutants of Saccharomyces cerevisiae that are defective in microtubule-mediated processes. Mol Cell Biol 10: 223-234.

Jakubowska A, Korona R (2012) Epistasis for growth rate and total metabolic flux in yeast. PLoS One 7: e33132.

Jorgensen P, Nishikawa JL, Breitkreutz BJ, Tyers M (2002) Systematic identification of pathways that couple cell growth and division in yeast. Science 297: 395-400.

Ludewig G, Williams JM, Li Y, Staben C (1994) Effects of pentamidine isethionate on Saccharomyces cerevisiae. Antimicrob Agents Chemother 38: 1123-1128.

Murata Y, Watanabe T, Sato M, Momose Y, Nakahara T, Oka S, Iwahashi H (2003) Dimethyl sulfoxide exposure facilitates phospholipid biosynthesis and cellular membrane proliferation in yeast cells. $J$ Biol Chem 278: 33185-33193.

Newton BA (1957) The mode of action of phenanthridines: the effect of ethidium bromide on cell division and nucleic acid synthesis. I Gen Microbiol 17: 718-730.

Pagani MA, Casamayor A, Serrano R, Atrian S, Arino J (2007) Disruption of iron homeostasis in Saccharomyces cerevisiae by high zinc levels: a genome-wide study. Mol Microbiol 65: 521-537.

Serrano R, Bernal D, Simon E, Arino J (2004) Copper and iron are the limiting factors for growth of the yeast Saccharomyces cerevisiae in an alkaline environment. J Biol Chem 279: 19698-19704.

Sirisattha S, Momose Y, Kitagawa E, Iwahashi H (2004) Toxicity of anionic detergents determined by Saccharomyces cerevisiae microarray analysis. Water Res 38: 61-70.
Steinmetz LM, Scharfe C, Deutschbauer AM et al. (2002) Systematic screen for human disease genes in yeast. Nat Genet 31: 400-404.

Stocklein W, Piepersberg W (1980) Binding of cycloheximide to ribosomes from wild-type and mutant strains of Saccharomyces cerevisiae. Antimicrob Agents Chemother 18: 863-867.

Venables P, Russell AD (1975) Nystatin-induced changes in Saccharomyces cerevisiae. Antimicrob Agents Chemother 7: 121-127.

Verduyn C, Postma E, Scheffers WA, Van Dijken JP (1992) Effect of benzoic acid on metabolic fluxes in yeasts: a continuous-culture study on the regulation of respiration and alcoholic fermentation. Yeast 8: 501-517.

Wanke V, Cameroni E, Uotila A, Piccolis M, Urban J, Loewith R, De Virgilio C (2008) Caffeine extends yeast lifespan by targeting TORC1. Mol Microbiol 69: 277-285.

Warringer J, Ericson E, Fernandez L, Nerman O, Blomberg A (2003) High-resolution yeast phenomics resolves different physiological features in the saline response. Proc Natl Acad Sci USA 100: 15724 15729.

Wemmie JA, Steggerda SM, Moye-Rowley WS (1997) The Saccharomyces cerevisiae AP-1 protein discriminates between oxidative stress elicited by the oxidants H2O2 and diamide. I Biol Chem 272: 7908-7914.

Yasokawa D, Murata S, Kitagawa E, Iwahashi Y, Nakagawa R, Hashido T, Iwahashi H (2008) Mechanisms of copper toxicity in Saccharomyces cerevisiae determined by microarray analysis. Environ Toxicol 23: 599-606.

Yasokawa D, Murata S, Iwahashi Y, Kitagawa E, Nakagawa R, Hashido T, Iwahashi H (2010) Toxicity of methanol and formaldehyde towards Saccharomyces cerevisiae as assessed by DNA microarray analysis. Appl Biochem Biotechnol 160: 1685-1698.

Zaman S, Lippman SI, Zhao X, Broach JR (2008) How Saccharomyces responds to nutrients. Annu Rev Genet 42: 27-81. 